\title{
Photon detection by a cryogenic InSb detector
}

\section{$\operatorname{AUTHOR}(\mathrm{S})$ :}

Kanno, Ikuo; Hishiki, Shigeomi; Sugiura, Osamu; Xiang, Ruifei; Nakamura, Tatsuya; Katagiri, Masaki

\section{CITATION:}

Kanno, Ikuo ... [et al]. Photon detection by a cryogenic InSb detector. REVIEW OF SCIENTIFIC INSTRUMENTS 2005, 76(2): 023102.

\section{ISSUE DATE:}

2005-02

URL:

http://hdl.handle.net/2433/39811

\section{RIGHT:}

Copyright 2005 American Institute of Physics. This article may be downloaded for personal use only. Any other use requires prior permission of the author and the American Institute of Physics. 


\title{
Photon detection by a cryogenic InSb detector
}

\author{
Ikuo Kanno and Shigeomi Hishiki \\ Graduate School of Engineering, Kyoto University, Sakyo, Kyoto 606-8501, Japan
}

Osamu Sugiura and Ruifei Xiang

Graduate School of Science and Engineering, Tokyo Institute of Technology, Meguro, Tokyo 152-8552, Japan

Tatsuya Nakamura and Masaki Katagiri

Japan Atomic Energy Research Institute, Tokai, Ibaraki 319-1195, Japan

(Received 25 October 2004; accepted 1 November 2004; published online 5 January 2005)

Gamma rays of ${ }^{241} \mathrm{Am}$ were measured by a Schottky type undoped $\mathrm{InSb}$ detector at $4.2 \mathrm{~K}$. Although a photopeak was not observed, the signals of gamma rays were clearly separated from noises.

(c) 2005 American Institute of Physics. [DOI: 10.1063/1.1835632]

\section{INTRODUCTION}

Compound semiconductor InSb has many favorable features as a substrate for radiation detectors: the smallest bandgap energy, $0.165 \mathrm{eV}$, among developed semiconductor materials, high atomic numbers, In:49, Sb:51, high density, $5.78 \mathrm{~g} \mathrm{~cm}^{-3}$, and high mobilities of electrons and holes, 78000 and $750 \mathrm{~cm}^{2} \mathrm{~V}^{-1} \mathrm{~s}^{-1}$ at $77 \mathrm{~K}$, respectively. The bandgap energy gives us the energy resolution better than that of Ge by a factor of at least two, assuming the Fano factor comparable to that for Ge. The atomic numbers and the density predict 6.5 times higher photon absorption probability than that of $\mathrm{Ge}$, provided that the photon absorption probability is proportional to $Z^{4} \rho$, where, $Z$ is the atomic number and $\rho$ the density. The high mobilities of electrons and holes of InSb bring us high efficiency in photon detection: comparing the performance of an InSb detector to a CdTe detector with the same resistivity, thicker depletion layer is expected due to higher mobilities, although photon absorption efficiency of InSb is nearly the same as with that of CdTe. If we could utilize InSb as a substrate for a radiation detector, detection of light elements such as $\mathrm{Be}$ and $\mathrm{Li}$ becomes possible with measuring characteristic $\mathrm{x}$ rays.

McHarris pointed out the advantages of InSb as the substrate of a radiation detector in $1986 .{ }^{1}$ There were, however, no activities on radiation detectors made of InSb before our work on the rectifying characteristics of an InSb device. ${ }^{2}$

Recently, we fabricated Schottky type and $p n$ junction type detectors with $p$-InSb, and Schottky type detectors with undoped InSb. They were operated at the temperature from 0.6 to $115 \mathrm{~K}$ for the detection of alpha particles of ${ }^{241} \mathrm{Am} .{ }^{3,4}$ Although the depletion layers of these InSb detectors were not thick enough for $5.5 \mathrm{MeV}$ alpha particles to deposit all of their energies, pulse heights due to alpha particles were observed, being clearly separated from electric noises.

As an application of the InSb detector, neutron detection at the operating temperature below $4.2 \mathrm{~K}$ was carried out using the nuclear reaction of $n+{ }^{3} \mathrm{He} \rightarrow p+t$. The energy spectra of protons $(574 \mathrm{keV})$ and tritons $(191 \mathrm{keV})$ were clearly measured by the undoped InSb detector with the Schottky electrode of $3 \mathrm{~mm}$ in diameter. ${ }^{5}$
Our purpose of developing InSb detectors is the detection of photons. In case of measuring gamma rays of ${ }^{241} \mathrm{Am}$, $59 \mathrm{keV}$ in energy, we have to reduce the electric noise to 1/10: as described above, the measurements of the protons with $574 \mathrm{keV}$ in energy were successfully carried out. To realize this condition, we fabricated $\mathrm{InSb}$ detectors with smaller electrodes to reduce the electric capacitances of them.

In this article, the fabrication method of the InSb detector, the current-voltage curves of it as a function of operating temperature, and the energy spectrum of measured gamma rays are described.

\section{EXPERIMENT}

\section{A. Device fabrication and current-voltage curves}

The employed InSb wafer was undoped with diameter of 2 in. and thickness of $0.5 \mathrm{~mm}$ (Sumitomo Electric Industries, Japan). This undoped wafer was $n$-type by the polarity of preamplifier output pulses in the alpha particle measurements. ${ }^{6}$ The wafer was cut to the dimensions of nearly $5 \mathrm{~mm} \times 7 \mathrm{~mm}$. Both sides of the InSb substrate were etched using a mixture of nitric and lactic acids (1:10) for $5 \mathrm{~min}$. On one of the InSb substrate surfaces, Au-Pd (60\%:40\%) alloy was deposited by heat evaporation with a thickness of $4 \mathrm{~nm}$ as a Schottky contact. After this process, an electrode area with the diameter of $1.5 \mathrm{~mm}$ was defined by a photoresist mask, and the rest of the surface was etched out to fabricate a mesa electrode. The height of the mesa was nearly $10 \mu \mathrm{m}$. Finally, the processed wafer was mounted on

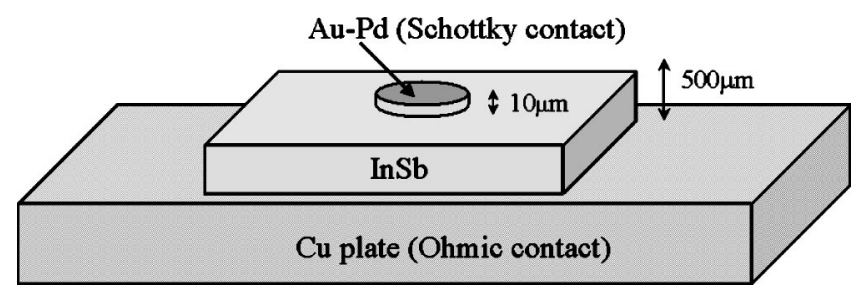

FIG. 1. Schematic drawing of an InSb detector. 


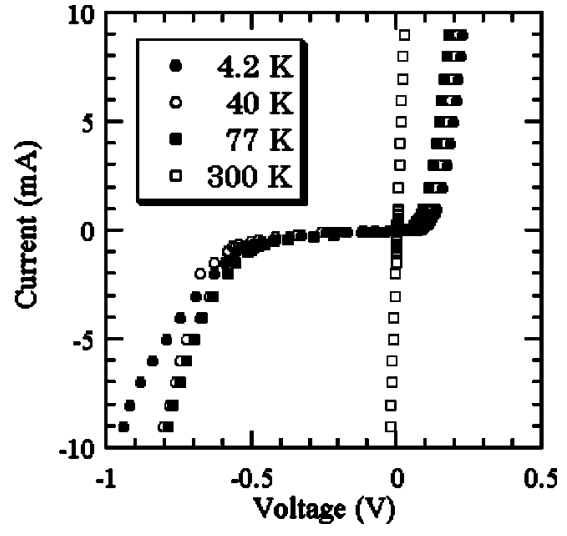

FIG. 2. Current-voltage curves of the InSb detector. The operating temperatures are shown in the figure.

a Cu plate by In solder, having an Ohmic contact on the back surface of the InSb wafer. Figure 1 shows a schematic drawing of the InSb detector.

The fabricated $\mathrm{InSb}$ detector was mounted on a probe and inserted into a liquid $\mathrm{He}$ dewar for current-voltage $(I-V)$ measurements. Measured $I-V$ curves are shown in Fig. 2 as a function of temperature. The non-linearity of $I-V$ curve was observed even at $77 \mathrm{~K}$.

\section{B. Gamma-ray measurement}

The $\mathrm{InSb}$ detector was mounted on a $0.3 \mathrm{~K}$ stage of a refrigerator (Infrared Co.). The InSb detector was exposed by gamma rays of an electrodeposited ${ }^{241} \mathrm{Am}$ source in the atmosphere through two Be windows on the outer and inner vessels of the refrigerator, respectively.

Figure 3 shows the block diagram of the electronic circuit for the measurements of the gamma rays. The output pulses of a preamplifier and a main amplifier were observed by a digital storage oscilloscope (DSO), and the pulse height spectra were measured by a multichannel analyzer (MCA).

The resistance of the preamplifier was changed from 100 to $2.2 \mathrm{M} \Omega$, because the resistance of the InSb detector was smaller by some orders of magnitude than the ones of general Si detectors. Measurements were, however, carried out without applying any bias voltage: large electric noises

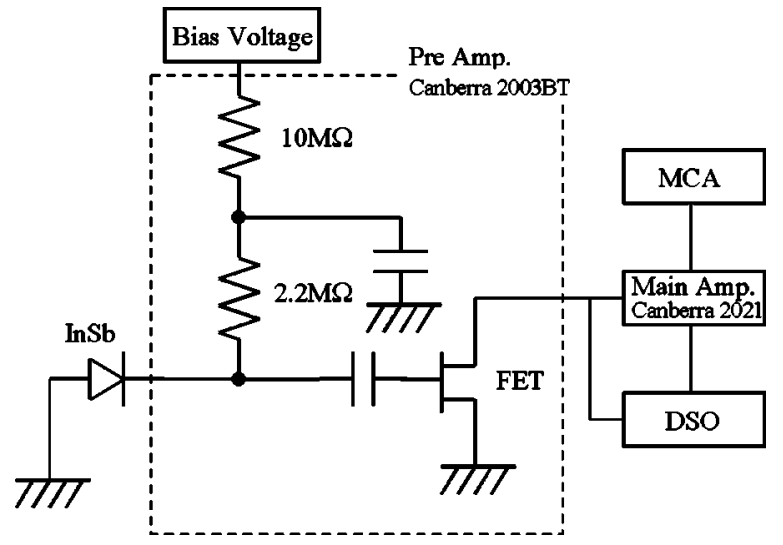

FIG. 3. Block diagram of the electronic circuit for the measurements of gamma rays. DSO: digital storage oscilloscope, MCA: multichannel analyzer.

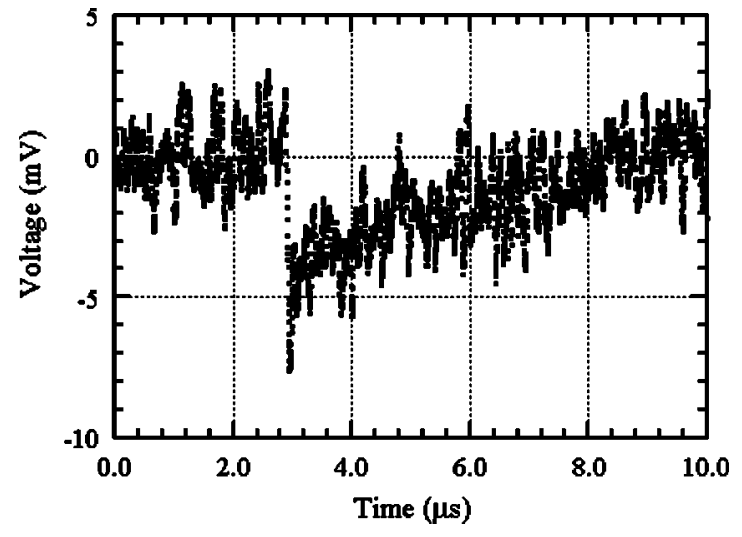

FIG. 4. Preamplifier output pulses of a gamma ray of ${ }^{241} \mathrm{Am}$.

were observed when the bias voltage was applied, as was described in the previous papers (Refs. 3-6). Both the foreground and background measurements were performed for $5 \mathrm{~min}$ and repeated several times. Measurements were performed at $4.2 \mathrm{~K}$.

\section{DISCUSSION}

An example of preamplifier output pulse is shown in Fig. 4. The typical amplitude of the preamplifier output was some $\mathrm{mV}$ and $10 \%-90 \%$ rise time was nearly $50 \mathrm{~ns}$. This rise time is shorter than the one of alpha particle measurements in Ref. 6 by one order of magnitude. The energy spectrum of the gamma rays is shown in Fig. 5, as well as the background. Although the photopeak could not be observed, the gamma ray signals were clearly separated from noises. The sources of noise are not studied in detail, however, the leak current of the detector might be the main noise.

In case of the energy of gamma rays being converted into electrons by photoelectric effect, the energy of photoelectrons is almost the same as that of primary gamma rays, i.e., $59 \mathrm{keV}$. The range of electrons with $59 \mathrm{keV}$ in $\mathrm{InSb}$ substrate is estimated as nearly $20 \mu \mathrm{m}^{7}$ On the other hand, the depletion layer thickness of the undoped InSb detector without applying bias voltage was $9 \mu \mathrm{m}$. ${ }^{6}$ Most of the photoelectrons escaped from the depletion layer with depositing their energy partly. For the measurement of a photopeak, the

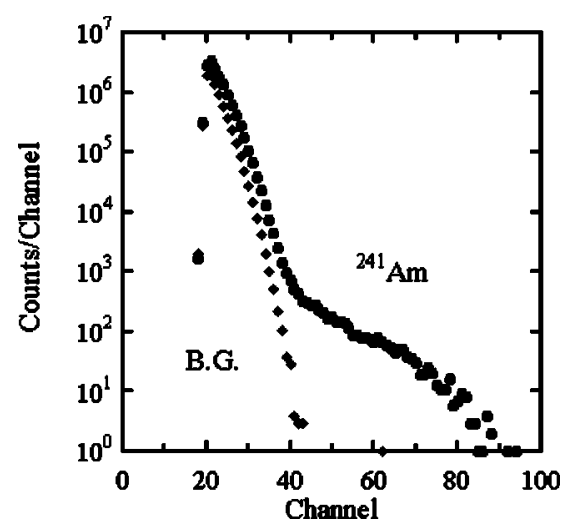

FIG. 5. Measured energy spectra of ${ }^{241} \mathrm{Am}$ gamma rays and background (BG). 
thickness of the depletion layer should be increased. The growth of high quality InSb crystals and the resulting decrease of leak current are necessary.

\section{ACKNOWLEDGMENTS}

This work was supported by the Grants-in-Aid for Scientific Research, Japan Society for Promotion of Science, and the Iketani Science and Technology Foundation.

${ }^{1}$ W. C. McHarris, Nucl. Instrum. Methods Phys. Res. A 242, 373 (1986).
${ }^{2}$ I. Kanno, F. Yoshihara, O. Sugiura, T. Nakamura, and M. Katagiri, Proceedings of the Ninth International Workshop on Low Temp. Detectors CP-605, 185 (2002).

${ }^{3}$ I. Kanno, F. Yoshihara, R. Nouchi, O. Sugiura, T. Nakamura, and M. Katagiri, Rev. Sci. Instrum. 73, 2533 (2002).

${ }^{4}$ I. Kanno, F. Yoshihara, R. Nouchi, O. Sugiura, Y. Murase, T. Nakamura, and M. Katagiri, Rev. Sci. Instrum. 74, 3968 (2003).

${ }^{5}$ T. Nakamura, M. Katagiri, Y. Aratono, I. Kanno, S. Hishiki, O. Sugiura, and Y. Murase, Rev. Sci. Instrum. 75, 340 (2004).

${ }^{6}$ S. Hishiki, I. Kanno, O. Sugiura, R. Xiang, T. Nakamura, and M. Katagiri, Rev. Sci. Instrum. (submitted).

${ }^{7}$ M. J. Berger, J. S. Coursey, and M. A. Zucker, NISTIR 4999 (2000). 